\title{
Proposta de Sistema Tutor Inteligente para Engenharia Geotécnica
}

\author{
Raymundo Carlos Machado Ferreira Filho ${ }^{1}$ \\ Nilo César Consoli** \\ Rosa Maria Vicari*** \\ Fernando Schnaid* \\ Ricardo Azambuja Silveira**
}

Resumo: este artigo descreve o planejamento e produção de um conjunto de atividades relacionadas à estruturação do conhecimento envolvido no domínio da Geotecnia, área da Engenharia Civil. O projeto propõe uma ontologia para desenvolvimento de um modelo conceitual e uma base de conhecimento que serão utilizados em um Sistema Tutor Inteligente, uma sub-área da Inteligência Artificial. Para a modelagem da base de conhecimento foi adotada a API Java Protégé-2000 desenvolvida pelo grupo de pesquisa em Informática Médica da Faculdade de Medicina da Universidade de Stanford (Califórnia, Estados Unidos). Integrada à base de conhecimento tem-se um repositório de recursos educacionais descritos através de metadados do padrão Dublin Core. A arquitetura proposta para esta aplicação WEB é composta pela plataforma de comunicação de agentes FIPA-OS, pelo servidor de base de dados MySQL, pela plataforma J2SE e pelo container Jakarta Tomcat . Serão apresentados neste artigo os resultados preliminares da arquitetura proposta.

Palavras-chave: Inteligência Artificial, Sistemas Tutores, Ciências Cognitivas, Tecnologia Educacional, Objetos de Aprendizado.

\section{Proposal of Intelligent Tutoring System for Geotechnical Engineering}

Abstract: This article describes the project and execution of several activities concerned to knowledge structure involving Geotechnical Engineering, a Civil Engineering area. It proposes ontology to develop a conceptual model and a knowledge basis that will be used in an Intelligent Tutor System. To model the knowledge basis, the API Java Protégé-2000 developed by Stanford Medical Informatics at the Stanford University School of Medicine (California, United States) research group was chosen. Within this knowledge basis there is a variety of educational facilities described through Dublin Core metadata. The proposed architecture to this application is composed by communication agents FIPA-OS, database server MySQL, J2SE and Jakarta Tomcat

\footnotetext{
${ }^{1}$ Doutorando do Programa de Pós-graduação em Informática na Educação (PGIE) - Universidade Federal do Rio Grande doSul-paka@ufrgs.br.

** Professor do Departamento de Engenharia Civil (PPGEC) da Universidade Federal do Rio Grande do Sul, PhD. (Concordia University, Canadá, 1991). Pesquisador do CNPq. Consultor "AD HOC" do CNPq e FAPESP consoli@ufrgs.br.

*** Professora adjunta do Departamento de Informática da Universidade Federal do Rio Grande do Sul, graduação e pós-graduação (CPGCC) desde 1987. Coordenadora do curso de Pós-graduação em Informática na Educação da UFRGS desde 1997. Pós-doutorado na Universidade de Leeds, UK, 1997. Grupo CBLU - Computer Base Learning Unit. rosa@inf.ufrgs.br.

***** Professor do Departamento de Engenharia Civil (PPGEC) da Universidade Federal do Rio Grande do Sul, PhD. (Oxford University, U.K., 1990). Pesquisador do CNPq; Coordenador do Laboratório de Ensaios Geotécnicos e Geoambientais (LEGG); Professor visitante das Universidades de Oxford, U.K.; Newcastle, AUS; Universidade Nacional del Nordeste, ARG, Universidad de la Republica, UR - fernando@ufrgs.br.

Professor do Departamento de Matemática, Estatística e Computação da Universidade Federal de Pelotas. Doutor em Ciência da Computação- UFRGS - rsilv@ ufpel.tche.br.

V.2 $\mathrm{N}^{\circ} 2$, Novembro, 2004
} 
container. The first results of the proposed knowledge structure will be shown in this article.

Keywords: Artificial Intelligence, Tutoring Systems, Cognitive Sciences, Educational Technology, Learning Objects.

\section{Introdução}

Um dos principais focos da equipe do NMEAD é a pesquisa relacionada ao uso de novas Tecnologias da Informação e Comunicação (TICs) aplicadas ao processo educacional. Do Grupo IATE, além da aplicação das TICs, o uso de técnicas de Inteligência Artificial em sistemas de ensino baseados na WEB, conhecidos como Ambientes Inteligentes de Ensino (ILE - Intelligent Learning Environment) e o compartilhamento e re-uso de objetos educacionais. Neste artigo, descreve-se e analisase o processo de produção de um projeto de aplicação dessas tecnologias educacionais, relacionando-as a objetivos e estratégias pedagógicas, para o ensino de Engenharia, em particular no curso de Engenharia Civil da Escola de Engenharia da UFRGS, com participação do Grupo IATE.

\section{Considerações sobre ambientes educacionais baseados na WEB e o papel educacional das TICs}

Segundo Moran (1998), construir conhecimento, hoje, significa compreender todas as dimensões da realidade, captando e expressando essa totalidade de forma ampla e integral. Suas pesquisas mostram que o processo de construção do conhecimento é mais bem desenvolvido quando um determinado objeto é acessado de forma conectada, interrelacionada, com seus múltiplos fatores interferentes, a partir de múltiplos pontos de vista, múltiplos caminhos, integrados da forma mais rica possível. Esta e outras idéias dessa natureza, que proliferaram na área de pesquisa educacional ao longo da última década, levam a considerar a importância da WEB como instrumento de acesso a informações e de construção de um conhecimento com a referida natureza múltipla, multifacetada. Trata-se de um instrumento, portanto, que não pode ser negligenciado no que diz respeito a sua utilização como meio para o ensino a distância (EAD) e semipresencial, uma vez que possibilita esse tipo de construção de conhecimento não linear e inter-relacionado. Moran (idem) diz ainda que se deve buscar nas áreas da Pedagogia e da Psicologia a fundamentação para a utilização educacional da rede, através de teorias e estratégias pedagógicas que fundamentem o processo de aprendizado mediado por computador. Teorias e estratégias que se agregam, através da rede, às possibilidades abertas pelos conhecimentos das Ciências da Computação e da Comunicação Social.

Cada mídia ou ferramenta de interação/cooperação oferecida ao aluno no ambiente virtual deverá contar sua respectiva equação do que Alava (Alava e outros, 2000) identifica como uma potencialidade da educação em ambiente virtual: o binômio método/mídia. Isso significa que cada tecnologia escolhida pelo planejador/professor deve agregar ao universo pedagógico sua própria contribuição. Um vídeo, cuja característica normalmente costuma estar associada a uma postura de simples recepção do aluno, pode ter sido planejado para mostrar a ele os detalhes do mundo real, da prática profissional à qual o conteúdo se refere, através da documentação de imagens e, se possível, a identificação da relação existente entre essa realidade e o universo de conteúdos.

O ambiente planejado para comportar o conteúdo de Geotecnia tem por finalidade atender a essa base didático-pedagógicas. Os alunos deverão ser envolvidos em um ambiente estimulante, criativo e interativo, composto de um repositório de objetos 
educacionais como clipes de vídeo, animações, simulações, emulações da realidade, associados a outros elementos de apoio, como textos e indicação de endereços na WEB relacionados ao tema, além de oferecer um sistema especialista (que será brevemente descrito a seguir, assim como a arquitetura proposta) incorporado ao ILE. Tanto quanto disponibilizar conteúdos do domínio, o ambiente (destinado prioritariamente a alunos de graduação e pós do curso de Engenharia Civil) tem também como meta, testar as estratégias desenvolvidas, para justificar (ou não) a expansão da mesma idéia para outras áreas. Em especial, para testar a chamada natureza interativa da Internet, oferecendo aos estudantes acesso remoto para aplicações e exercícios com várias possibilidades de acesso, por área de interesse dentro do domínio, por tipos de mídia específico ou por assunto.

\section{Sistemas Especialistas apoiando o processo de tomada de decisão}

De acordo com Rezende (2003) existem quatro tipos de conhecimento: o declarativo (descritivo e genérico), o procedural (prescritivo, determinístico, ordinário), de senso comum (composição dos dois anteriores) e o heurístico (obtido através de experiência pessoal que se transformam em regras práticas). A aquisição do conhecimento heurístico é a mais difícil e a que demanda o maior esforço do engenheiro do conhecimento e um problema em engenharia de software (Garcia, 2003; Flores, 2003; Azevedo; 1999). Mas a aquisição de conhecimento em um SE se restringe a domínios de conhecimento bem definidos e delimitados.

A tecnologia dos Sistemas Especialistas pode ser empregada para a conservação, organização e manutenção do conhecimento, particularmente do conhecimento heurístico. Ela possibilita o emprego de computadores para auxiliar na tomada de decisões. Além disso, essa tecnologia permite formalizar a sistematização do conhecimento prático existente em um domínio específico do conhecimento. A Geotecnia é uma área do conhecimento especialmente propícia para utilizar a tecnologia dos SE (Rezende et al., 2003; Flores, 2003; Azevedo; 1999), dada a natureza heurística do problema da escolha do tipo de fundação, acrescida da limitação do tempo disponível de uma disciplina, da pouca experiência na solução de problemas reais por inúmeros professores e da dificuldade dos especialistas em explicitar o conhecimento heurístico, muitas vezes inexistente no processo de ensino-aprendizagem. A etapa de desenvolvimento do SE que está sendo adotado já foi cumprida por Azevedo (1999) e foi amplamente descrita por ele sua tese de doutorado e a proposta é dar continuidade ao projeto desenvolvendo um aplicativo WEB para EAD e apoio a cursos presenciais.

O SE desenvolvido por Azevedo trata o conhecimento heurístico da prática regional de engenharia de fundações que tem um enorme potencial de utilização tanto acadêmico quanto comercial. Uma das justificativas de desenvolvimento do SE é tornar permanentemente acessível o conhecimento nas atividades do usuário. Contudo, Azevedo como ferramenta de produção a Shell Kappa-PC, que apesar de permitir uma variedade de estruturas de dados e de controle, não está mais disponível no mercado e todo o trabalho de doutoramento de Azevedo está sem utilidade, apesar de todo o potencial.

Outro problema é que é necessário que a Shell esteja instalada no computador do usuário para que o SE possa ser aberto e utilizado. Mesmo que a Shell ainda existisse no mercado, teríamos a dificuldade de integração com aplicativos WEB utilizados em ambientes de EAD ou apoio a cursos presenciais. Para realização do propósito de disponibilização do SE integrado ao ILE adotou-se a API Java Protégé-2000, um editor de ontologias e bases de conhecimento que permite criar classes Java, slots, restrições e regras para os slots, relações entre as classes e as propriedades destas relações. A 
manipulação e inferências na base de conhecimento serão realizadas por Agentes que estarão em uma camada intermediária entre a interface para o usuário e a própria base utilizando os recursos da plataforma de comunicação de agentes FIPA-OS.

Utilizando-se a API Protégé-2000 será criada uma ontologia que representa o domínio do conhecimento abordado e desenvolver um novo motor de inferência baseado nas regras e na base de conhecimento extraídos do SE de Azevedo. Esta nova arquitetura está de acordo com as recomendações atuais para desenvolvimento de sistemas baseados em conhecimento (Rezende, 2003), onde motor de inferência, base de conhecimento, interface com o usuário e demais componentes do sistema são implementados de forma integrada, porém com a possibilidade de incrementar cada um de forma independente de acordo com a demanda, como por exemplo, inferir novas regras e novo conhecimento ao sistema.

\section{Objetos educacionais e metadados}

Outro recurso que estará integra ao ILE é o repositório de objetos educacionais. Objetos educacionais podem ser descritos como qualquer recurso utilizado para apoio ao processo de aprendizagem. Este termo geralmente aplica-se a materiais educacionais planejados e produzidos com metodologia orientada a objetos (Tarouco et alli, 2003). A idéia é que os objetos educacionais sejam construídos em unidades básicas e que possam ser combinadas formando conjuntos instrucionais para serem utilizados nos mais diversos contextos. A produção de material educacional exige tempo, recursos financeiros e recursos humanos e existe uma demanda no sentido de otimizar a aplicação destes recursos. Desta forma o compartilhamento e o reuso de objetos educacionais se torna justificável e sua adoção é recomendada pelos autores.

Uma grande quantidade de recursos digitais já foram produzidos a partir de projetos realizados pelos grupos de pesquisa IATE e NMEAD. Este conjunto de materiais é composto de vídeos, imagens, ilustrações, animações e artigos relacionados ao domínio e compõem o repositório de objetos educacionais e pode ser acessado pelo endereço http://iate.ufrgs.br:8080/patologias. Adicionalmente à questão da produção de material educacional aparece a necessidade de catalogação e descrição dos recursos educacionais produzidos. O esforço de definição de padrões para catalogação de objetos surgiu do grande volume de informações disponibilizadas na Internet e da dificuldade quanto à localização e recuperação das informações buscadas pelos usuários da rede. A partir dessas dificuldades, as ferramentas apropriadas à organização dessa informação tem sido melhoradas, bem como os padrões que estão sendo empregados para sua catalogação. Instituições que trabalham na definição de padrões têm trabalhado neste sentido. É o caso do IEEE (1484.12.1-2002 Standard for Learning Object Metadata) e ISO (SC 36 WG 2 - Information Technology for Learning, Education and Training).

Os descritores, isto é, os diversos campos que descrevem um recurso eletrônico, seja ele educacional ou não, chama-se metadado, e são informações estruturadas que resumem, enriquecem ou complementam os objetos ou serviços referenciados, produzindo um potencial incremento de informação, auxiliando na eficiência e otimização dos mecanismos de busca e na recuperação de informações. Catalogar recursos eletrônicos é uma forma de organizar a informação e tornar mais fácil e rapidamente acessível o recurso. Conforme Souza (2000), a catalogação das informações com metadados permite a recuperação dessas informações disponibilizadas on-line. Em nossa proposta, os recursos educacionais estarão associados à base de conhecimento disponível no ILE.

O padrão de metadados escolhido para desenvolver a base de dados deste trabalho foi o Dublin Core (DC). Este padrão inclui dois níveis: simples e qualificado. O DC 
simples especifica 15 elementos e o nível qualificado inclui um elemento adicional e pode ser definido como sendo o conjunto de elementos de metadados planejado para facilitar a descrição de recursos eletrônicos (Hillmann, 2003). A expectativa é que, a partir de um sistema de catalogação on-line, professores e tutores sem conhecimento de catalogação e programação sejam capazes de usar o DC para descrição de recursos eletrônicos, tornando suas coleções mais visíveis pelos mecanismos de busca e sistemas de recuperação de informação, que pode ser tarefa de um agente inteligente, pelos sistemas de armazenamento de informação em geral, e pelos usuários do sistema, no caso os alunos dos cursos de Geotecnia. Além disso, pretende-se integrar os objetos educacionais relacionados ao tema deste projeto (vídeos, animações, simulações, gráficos, imagens, etc) já produzidos pelo NMEAD ao SE que esta sendo implementado no ILE.

\section{O ambiente inteligente de aprendizado: integração dos recursos educacionais}

Antes de tudo, cabe ressaltar que a plataforma de ensino/aprendizagem para Geotecnia, ainda sem agentes, está em fase final de implantação, dispondo do repositório de objetos educacionais descritos com o padrão Dublin Core (já mencionado anteriormente no item anterior deste artigo) e a base de dados que dá suporte ao funcionamento do sistema de gestão do ambiente, bem como de informações referentes a disciplinas. A plataforma pode ser acessada pelo endereço http://iate.ufrgs.br:8080/patologias, como também já foi mencionado. A arquitetura proposta para agentificação deste ambiente é a de um sistema multicamadas onde temos na camada mais profunda as bases de dados, a base de conhecimento, os descritores DC e o repositório de objetos educacionais. A plataforma FIPA-OS dá suporte à comunicação entre agentes em uma camada intermediária e na camada mais externa (ou superior) o container oferece recursos que permitem interação com o usuário e préprocessamento de informações, como, por exemplo, instanciar um agente ou armazenar e enviar valores de variáveis que serão usadas pela aplicação WEB ou pelos agentes do ILE. Esta arquitetura pode ser visualizada na Figura 1. As especificações da plataforma FIPA-OS estão disponíveis no repositório do site da instituição "the Foundation for Intelligent Physical Agents - FIPA" no endereço http://www.fipa.org/repository/index.html. As informações sobre a plataforma Java2 ${ }^{\mathrm{TM}}$ Standard Edition e sobre o container Tomcat estão disponíveis em http://java.sun.com e http://jakarta.apache.org, respectivamente. 


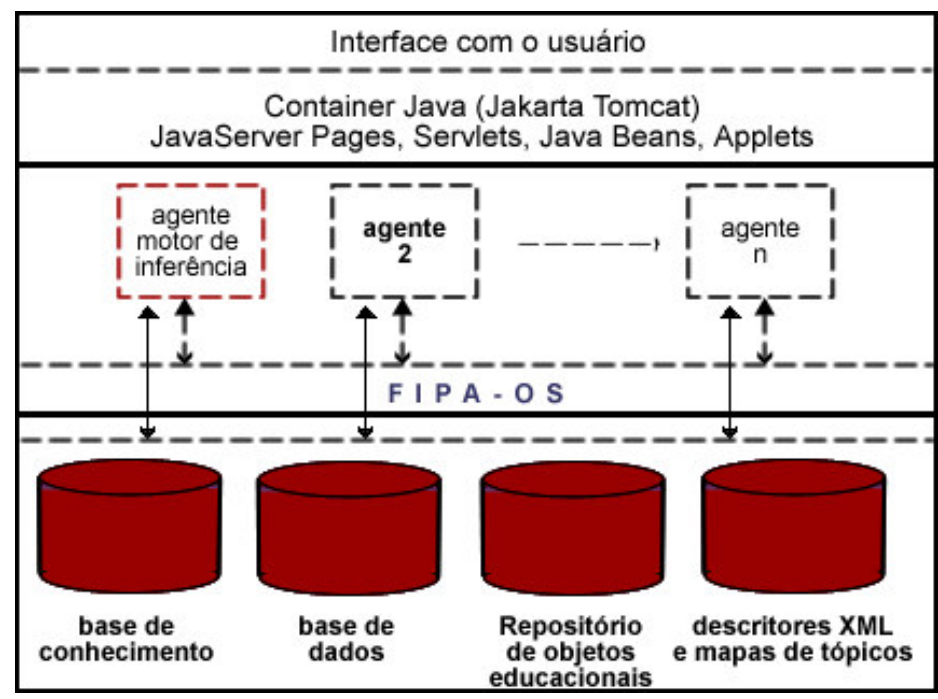

Figura 1: arquitetura proposta para o ILE

O que será descrito aqui é a migração e implementação de uma ontologia e base de conhecimento que será manipulada por um agente ou agentes (por ora, representado na Figura $1 \mathrm{com}$ o nome de agente motor de inferência). A estratégia pedagógica ainda não é tarefa desempenhada por agentes no ambiente de aprendizagem proposto. A implementação de ambientes de ensino baseados na WEB, seja para EAD ou para apoio ao ensino presencial, deve contemplar estratégias pedagógicas, mas esta discussão não será abordada neste artigo. Esta é uma questão de extrema relevância que está sendo discutida pelos grupos IATE e NMEAD com alguns resultados já publicados como podem ser vistos em Flores et al. (2003) e Vicari et al. (2003).

Este projeto está recuperando a base de conhecimento e as regras de produção do SE desenvolvido por Azevedo e implantando-o no ILE como uma tecnologia educacional para apoio ao aprendizado. O domínio de aplicação do SE é a escolha do tipo de fundação, subárea da Geotecnia, e teve como objetivo central verificar a possibilidade de desenvolver um modelo de conhecimento e implementá-lo num ambiente computacional, baseado nos princípios da engenharia do conhecimento, aplicados a área da engenharia de fundações, com a função de auxiliar na instrução e treinamento de alunos na tarefa de escolher os tipos de fundações tecnicamente adequados às condições impostas em problemas reais. Ainda segundo Azevedo:

"Os Sistemas Especialistas apresentam várias vantagens sobre os tradicionais métodos de ensino expositivo. Além de tornar a aprendizagem institucional mais realística e aumentar a motivação dos estudantes, permitem que o aluno, através da comunicação interativa com o sistema, torne-se um participante mais ativo no processo de aprendizagem, ao contrario dos ambientes tradicionais de sala de aula, onde o mesmo e um ser passivo" (Azevedo, 1999).

A tecnologia dos SE pode ser empregada para a conservação, organização e manutenção do conhecimento, particularmente do conhecimento heurístico. Ela possibilita o emprego de computadores para auxiliar na tomada de decisões em situações nas quais, até alguns anos atrás, não era possível a utilização de modelos computacionais. Além disso, essa tecnologia permite formalizar a sistematização do 
conhecimento prático existente em um domínio específico do conhecimento. O que se propõe é transformar o SE de Azevedo em um sistema de agentes implementados ILE. Dentro da arquitetura do ILE teremos o que Rezende (2003) chama de Núcleo do Sistema Baseado em Conhecimento (NSBC) como vemos na Figura 2. Nesta camada temos o(s) agente(s) responsável(s) pela interação com o usuário, que obtém informações através das sucessivas respostas dos usuários às perguntas realizadas através da interface do SE, pelo desenvolvimento do raciocínio baseado nas informações obtidas através das informações com o usuário e pelo conhecimento representado na base de conhecimento e que através de regras encontra a solução para as condições de contorno selecionadas pelo usuário e pela apresentação da resposta ao usuário através da interface.

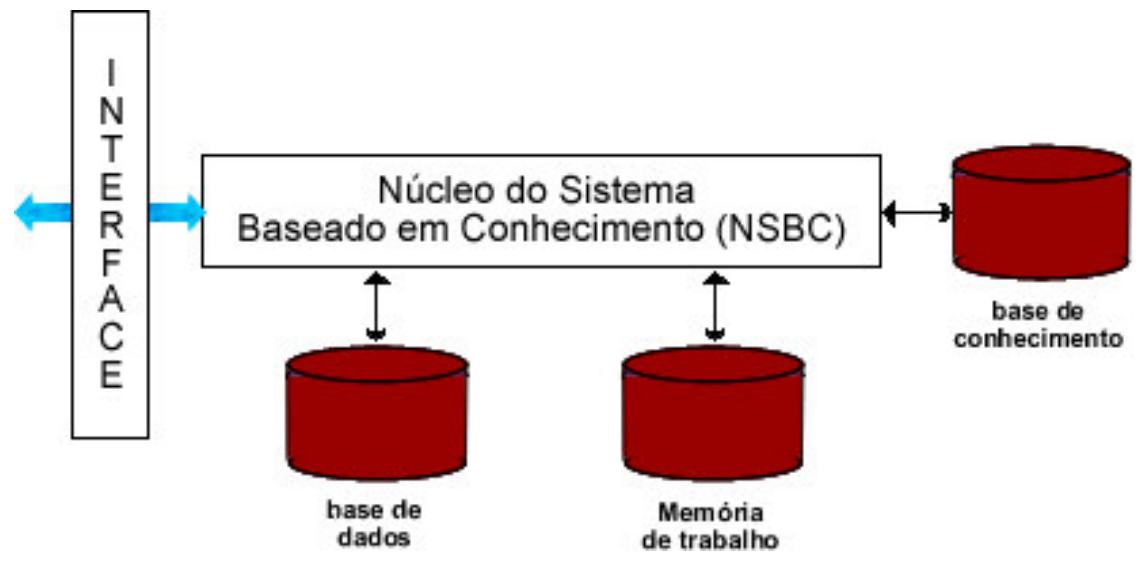

Figura 2: Representação da estrutura do sistema baseado em conhecimento que será integrado ao ILE

Cabe aqui uma breve descrição do funcionamento da versão do SE de Azevedo, do ponto de vista do usuário final (o aluno dos cursos de Geotecnia). Ao ser consultado, o sistema faz perguntas sobre: nível de carregamento, tipo de estrutura e condições de subsolo. Em função de cada resposta, o sistema apresenta a pergunta subseqüente. A solução oferece opções: comentário sobre a escolha; resumo de referências bibliográficas; relatório que pode ser impresso, contendo informações sobre a consulta (dados fornecidos, comentários, referências e outros, se for o caso). Algumas telas do SE original pode ser vistas na Figura 3, onde são apresentadas as telas de abertura, a de escolha do nível de carregamento, a de consulta sobre as informações do subsolo e a tela de resposta do sistema à consulta. 

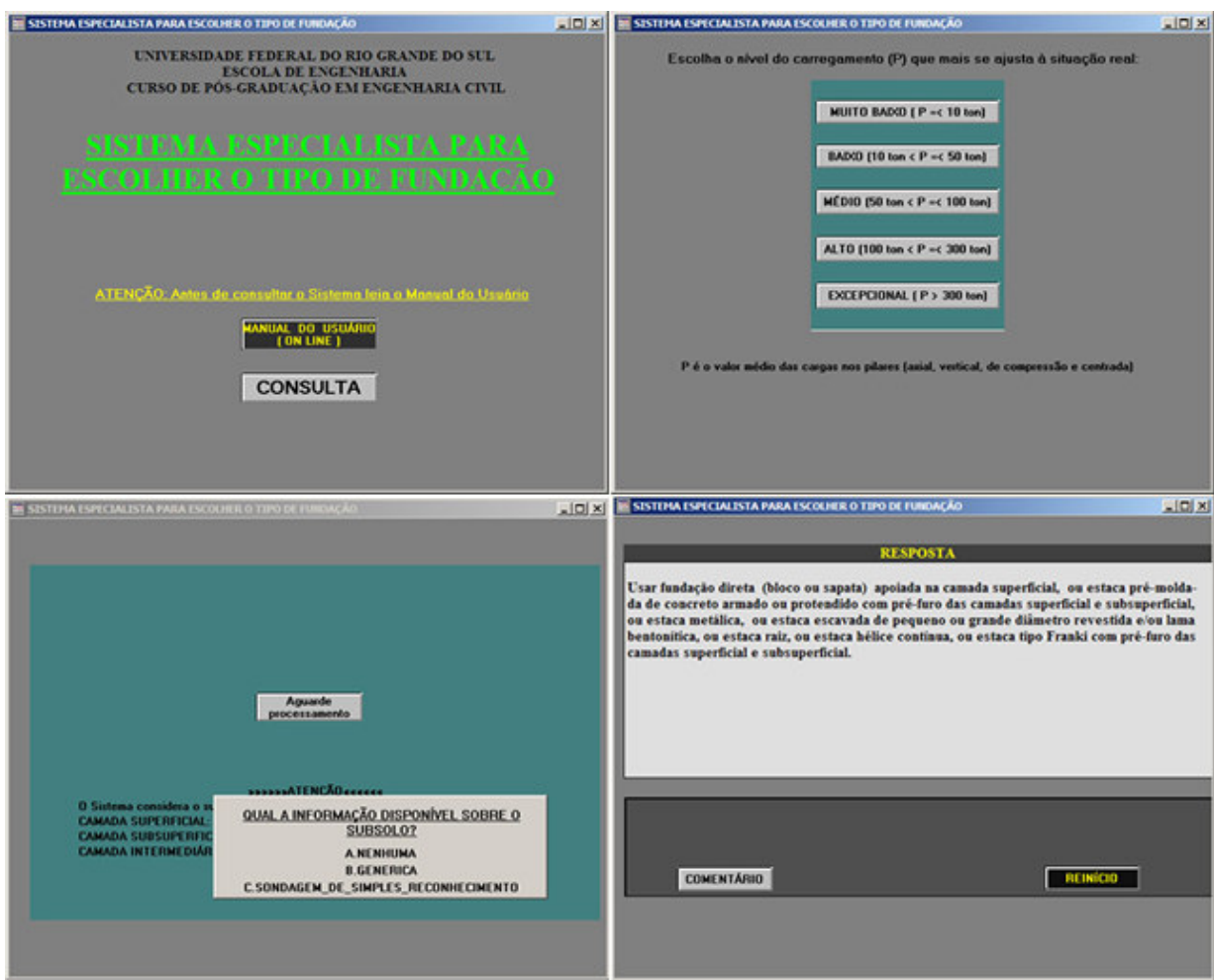

Figura 3: telas do SE de Azevedo, no sentido horário a partir da imagem do canto superior esquerdo, abertura, escolha do nível de carregamento, informações do subsolo e resposta à consulta.

A representação esquemática deste módulo que será implementado é mostrada na Figura 4, qual seja, a agentificação do SE de Azevedo incorporando-o ao ambiente de aprendizado já mencionado no início deste artigo caracterizando um ambiente inteligente de aprendizado (ILE).

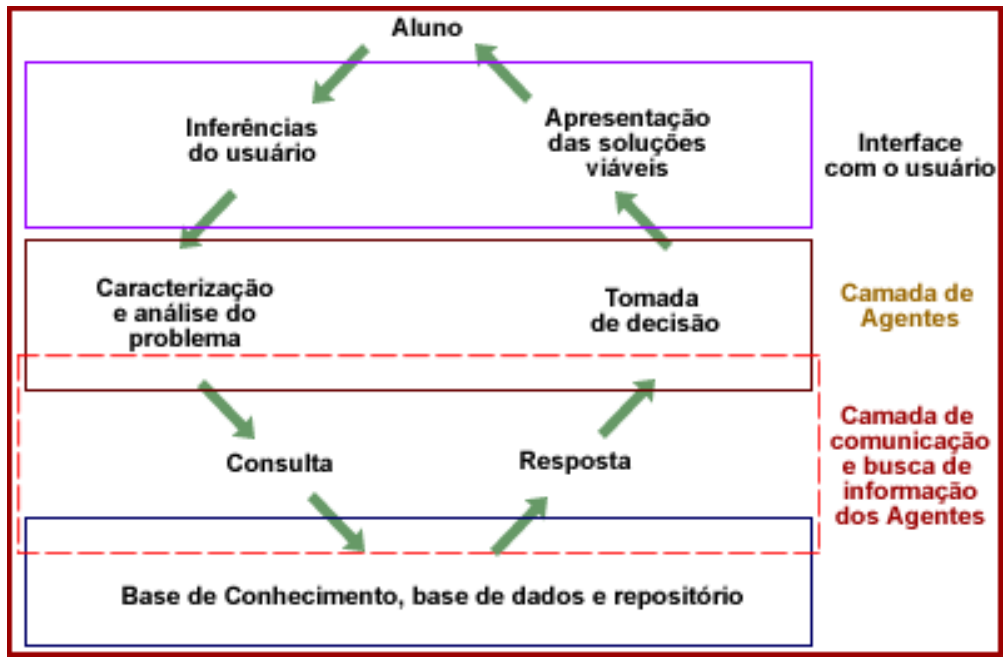

Figura 4: Representação do SE agentificado

\section{Implantação}

O SE de Azevedo foi desenvolvido, como já mencionado, na shell Kappa-PC e o aplicativo, em função da arquitetura própria software, tem seu motor de inferência, base 
de conhecimento, regras, interface e demais componentes encapsulados em um único arquivo binário. O software tinha por base a linguagem de programação LISP que não é a mais adequada para implementações WEB, mas que tinha vantagens para Azevedo conforme relato abaixo:

- Prototipagem rápida de SE;

- Usam estruturas de dados e conhecimento pré-definidas (menos flexibilidade, mas mais rapidez e tranquilidade para desenvolvedores);

- Menor necessidade de treinamento de desenvolvedores de SE (é mais simples construir um SE com "shell").

O projeto já cumpriu as etapas de estudo do trabalho de Azevedo e está na fase final de implantação do ambiente de aprendizado para Geotecnia. A etapa que está sendo iniciada é a programação das regras de produção, do motor de inferência e da base de conhecimento através da API Java Protege 2000. Desta fase resultará a estrutura descrita anteriormente neste artigo, onde teremos os componentes do SE independentes e implantados no ambiente WEB, todos eles implementados em Java e XML. Esta arquitetura possibilitará que mesmo depois de finalizada a implantação, continue possível se programar novas regras e inferir conhecimento novo à base. Estas tarefas são facilitadas pela adoção da API Protégé.

A implementação do SE no ambiente de aprendizado será acompanhado dos seguintes agentes artificiais, que estarão dispostos na camada de agentes (Figura 4). O papel de cada agente e a interação entre eles é descrito abaixo:

Agente Coletor de Dados (ACD): será responsável pela interação com o usuário, obtendo as informações do problema através da interação com a interface, que apresenta uma seqüência de perguntas que são respondidas pela escolha de opções previamente apresentadas na própria interface. Quando ativado pelo Agente Motor de Inferência, o ACD faz as perguntas ao usuário e valida as respostas baseando-se em regras préestabelecidas. Estas regras verificam a validade das respostas.

Agente Motor de Inferência (AMI): será responsável pelo desenvolvimento do raciocínio baseado nas informações obtidas pela comunicação com o Agente Coletor de Dados e pelo conhecimento representado na base de conhecimento.

Agente de Soluções e Conteúdo (ASC): ao buscar as soluções do problema em questão, o AMI necessita de algumas informações. Então o ACD é ativado de modo a questionar o usuário. Se o usuário questionar o porquê da pergunta, o ASC é ativado pelo ACD justificando a necessidade. Ao final do processo de inferência, o AMI chega às suas conclusões e as expõe ao usuário. A cada etapa intermediária de interação entre o sistema de agentes e o usuário é apresentada ao usuário a possibilidade de obter informações complementares através de conteúdo multimídia. O ASC busca conteúdo pertinente no repositório de objetos educacionais e apresenta-os ao usuário.

\section{Conclusões}

Pretende-se com este projeto, a melhoria da qualidade do ensino nas disciplinas da área de Geotecnia do curso de Engenharia Civil da UFRGS, e do ensino em Engenharia de modo geral, com possibilidade de compartilhamento, do conteúdo e do Ambiente de Ensino desenvolvido. O objetivo do ILE e do sistema baseado em conhecimento que esta sendo integrado, seja para o apoio às atividades presenciais ou a distância, é oferecer espaços onde os estudantes poderão ter experiências de aprendizagem individualizadas. Corroborando a idéia do uso de novas tecnologias na educação, Depover (2000, p. 153) argumenta que “... a escolha fundamental não se situa no fato de optar por tal tecnologia, mas na decisão de conceber uma seqüência ou um ambiente de aprendizagem segundo um modelo pedagógico adequado...". Ainda dentro desta mesma 
concepção, Vicari (2003 p.155) acrescenta: “Todo programa pode ser considerado um programa educacional, desde que utilize uma metodologia que o contextualize no processo de ensino-aprendizagem".

\section{Referências Bibliográficas}

ALAVA, S. e colaboradores. Ciberespaço e formações abertas: rumo a novas práticas educacionais?. Porto Alegre: Artmed, 2002.

AZEVEDO, S. L. Desenvolvimento de um protótipo de sistema especialista para escolha do tipo de fundação. Tese de doutorado - PPGEC/UFRGS. 1999.

DEPOVER, C. Um dispositivo de aprendizagem a distância baseado na partilha de conhecimento. In: Alava, S. e colaboradores. Ciberespaço e formações abertas: rumo a novas práticas educacionais?. Porto Alegre: Artmed, 2002.

DUBLIN CORE METADATA INITIATIVE (DCMI). DC type element schema. URI: http://dublincore.org/2000/03/13/dctype\# (acessado em 5 Junho de 2004)

DUBLIN CORE METADATA INITIATIVE (DCMI) Architecture Working Group: Expressing Qualified Dublin Core in RDF. URI: http://www.mathematik.uniosnabrueck.de/projects/dcqual/qual21.3.1/ (acessado em 7 Junho 2004)

FIPA: The foundation for Intelligent Physical Agents. Specifications. Disponível em http://www.fipa.org. Março de 2003.

FIPA-OS - FIPA Open Source. Available by HTTP in:<fipa-os.sourgeforge.net> Acessado em Março de 2003.

FLORES C. et al. Projeto AMPLIA - uso da informática na educação médica. Disponível em http://www.inf.ufrgs.br/ dflores/publicacoes/wim2003_AMPLIA.PDF. Acessado em Fevereiro de 2004.

HILLMANN, D. Using Dublin Core. 2003. Disponível em <http://dublincore.org/documents/usageguide/>. Acesso em 16/11/2003.

IEEE P1600.1 - STANDARD UPPER ONTOLOGY WORKING GROUP (SUO WG). http://suo.ieee.org/ acessado em 10 de junho de 2004.

KOLB, D. A. Experimental learning: experience as the source of learning and development. New Jersey: Prentice-Hall. 1984.

LANDRY, P. O sistema educativo rejeitará a Intenet? Ou as condições para uma boa integração das mídias nos dispositivos. In: Alava, S. e colaboradores. Ciberespaço e formações abertas: rumo a novas práticas educacionais?. Porto Alegre: Artmed, 2002.

MORAN, J. M. Ensino e aprendizagem inovadores com tecnologia. Revista Informática na Educação - PGIE/UFRGS. 2000.

MORAN, J. M. Mudanças na Comunicação Pessoal: gerenciamento integrado da comunicação pessoal, social e tecnológica. São Paulo: Paulinas, 1998.

PIAGET, J. Epistemologia Genética. São Paulo: Abril, 1978.

REZENDE, S. O. ET ALLI. Sistemas Baseados em Conhecimento. In: Sistemas Inteligentes: fundamentos e aplicações. Editora Manole. Barueri, SP. 2003.

ROSSETO, M.; NOGUEIRA, A.H. Aplicação de elementos metadados Dublin Core para descrição de dados bibliográficos on-line da biblioteca digital de teses da USP. Disponível em <www.sibi.ufrj.br/snbu/snbu2002/oralpdf/82.a.pdf>. Acesso em 13/02/2004.

SCHNAID, F.; TIMM, M. I.; ZARO, M. A.; FERREIRA FILHO R. C. M.; Rosa A. M. O. Mídia para Educação a Distância. In: VI WORKSHOP INFORMÁTICA NA EDUCAÇÃO, 2002a, Porto Alegre. 
SCHNAID, F.; TIMM, M. I.; ZARO, M. A.; FERREIRA FILHO R. C. M.. Multimídia e aulas interativas a distância: experiências de aplicação no ensino de engenharia Civil. In: VI WORKSHOP INFORMÁTICA NA EDUCAÇÃO, 2002b, Porto Alegre.

RUSSEL, T.; NORVIG P.. Inteligência Artificial. Rio de Janeiro: Elsevier, 2004.

TAROUCO, L. M. R.; FABRE M. J. M.; TAMUSIUNAS, F. R.. Reusabilidade de objetos educacionais. RENOTE - Revista Novas Tecnologias na Educação: II Ciclo de Palestras sobre Novas Tecnologias na Educação. Porto Alegre, RS, 2003.

THE PROTÉGÉ ONTOLOGY EDITOR AND KNOWLEDGE ACQUISITION SYSTEM. http://protege.stanford.edu acessado em 10 de junho de 2004.

VICARI, R. M.; GIRAFFA, L. M. M. Fundamentos de Sistemas Tutores Inteligentes. In: Barone, D.; et alii. Sociedades artificiais: a nova fronteira da inteligência nas máquinas. Porto Alegre: Bookman, 2003.

VICCARI, R. M. et al. A Multi-Agent Intelligent Environment for Medical Knowledge. Artificial Intelligence in Medicine, Elsevier Science B. V., v. 27, p. 335366, 2003.

WORLD WIDE WEB CONSORTIUM (W3C). Annotea Project. 31 May 2001. URI: http://www.w3.org/2001/Annotea/ (acessado em 7 Junho 2004)

WORLD WIDE WEB CONSORTIUM (W3C). Amaya Home Page - W3C's Editor/Browser. 23 April 2001. URI: http://www.w3.org/Amaya/ (acessado em 7 Junho 2004)

WORLD WIDE WEB CONSORTIUM (W3C) DATE AND TIME FORMATS. URI: http://www.w3.org/TR/NOTE-datetime (acessado em 7 Junho 2004)

DUBLIN CORE METADATA INITIATIVE (DCMI). An XML Encoding of Simple Dublin Core Metadata. URI: http://dublincore.org/documents/2000/11/dcmesxml/ (acessado em 23 Fevereiro de 2003)

WORLD WIDE WEB CONSORTIUM (W3C) RESOURCE DESCRIPTION FRAMEWORK (RDF). http://www.w3.org/RDF/ (acessado em 10 Junho de 2004) 